OPEN ACCESS

Edited by:

Ruiwen Zhang,

University of Houston, United States

Reviewed by:

Busisiwe Bhengu,

University of KwaZulu-Natal, South

Africa

Ye Huang,

China Academy of Chinese Medical

Sciences, China

${ }^{*}$ Correspondence:

Qiang Xu

fjksg@163.com

James Cheng-Chung Wei

jccwei@gmail.com

${ }^{+}$These authors have contributed equally to this work

Specialty section: This article was submitted to

Ethnopharmacology,

a section of the journal

Frontiers in Pharmacology

Received: 30 January 2021 Accepted: 04 May 2021

Published: 17 May 2021

Citation:

Xiao $Y-Z$, Ye Z-Z, Liang Y-T, Chen X-P, Wang $Y-H, X u Q$ and Wei JC-C (2021) Association Between Chinese Herbal

Medicine Therapy and the Risk of

Chronic Kidney Disease in

Gout Patients.

Front. Pharmacol. 12:661282.

doi: 10.3389/fphar.2021.661282

\section{Association Between Chinese Herbal Medicine Therapy and the Risk of Chronic Kidney Disease in Gout Patients}

\author{
Yan-Zhuan Xiao ${ }^{1,2 t}$, Zhi-Zhong Ye ${ }^{3 \dagger}$, Yuan-Tong Liang ${ }^{1,2 \dagger}$, Xin-Peng Chen ${ }^{3}$, \\ Yu-Hsun Wang ${ }^{4 t}$, Qiang $X u^{1,2 *}$ and James Cheng-Chung Wei $i^{5,6,7 *}$
}

${ }^{1}$ Department of Rheumatology, The First Affiliated Hospital of Guangzhou, University of Chinese Medicine, Guangzhou, China,

${ }^{2}$ The First Clinical Medical School, Guangzhou University of Chinese Medicine, Guangzhou, China, ${ }^{3}$ Department of Rheumatology, Shenzhen Futian Hospital for Rheumatic Diseases, Shenzhen, China, ${ }^{4}$ Department of Medical Research, Chung Shan Medical University Hospital, Taichung, Taiwan, ${ }^{5}$ Department of Allergy, Immunology and Rheumatology, Chung Shan Medical University Hospital, Taichung, Taiwan, Institute of Medicine, Chung Shan Medical University, Taichung, Taiwan, ${ }^{7}$ Graduate Institute of Integrated Medicine, China Medical University, Taichung, Taiwan

Background: Chinese herbal medicine ( $\mathrm{CHM})$ has been nationally and globally used in treating gout for over a millennium. The potential relationship between the incidence of chronic kidney disease (CKD) in gout patients and CHM therapy is unclear. Thus, this study aimed to provide some evidence regarding the relationship between $\mathrm{CHM}$ therapy and the occurrence of CKD in gout patients.

Methods: We used data from the National Health Insurance Research database (NHIRD) in Taiwan. In this population-based nested case-control study, all participants were identified by International Classification of Diseases, Ninth Revision (ICD-9). Conditional logistic regression was used to calculate the odds ratio (OR) of the risk of CKD in gout patients treated with $\mathrm{CHM}$ therapy.

Results: Data on 1718 gout patients with CKD and 1:1 matched 1718 gout patients without CKD were collected for analysis. The results showed that $\mathrm{CHM}$ therapy in gout patients did not increase the risk of developing CKD (adjusted OR $=1.01 ; 95 \%$ confidence interval $[\mathrm{Cl}]$ : 0.86-1.18; $p>0.05)$. Moreover, $\mathrm{CHM}$ therapy in gout patients for $>365$ days did not increase the incidence of CKD (adjusted OR $=1.30 ; 95 \% \mathrm{Cl}: 0.90-1.88 ; p=0.162$ ).

Conclusion: Traditional CHM therapy does not increase the incidence of CKD in gout patients.

Keywords: gout, chronic kidney disease, pharmacologic (drug) therapy, treatment, Chinese herbal medicine

\section{INTRODUCTION}

The incidence of gout is the highest among all types of arthritis (Kuo et al., 2015). According to the Global Health Data Exchange and the World Health Organization database, the global incidence and prevalence of gout have increased rapidly over the past 3 decades, with a 37 and $41 \%$ increase in its incidence and prevalence, respectively from 1992 to 2017 (Mattiuzz and Lippi, 2020). Clinical manifestations include severe joint pain, swelling, and elevated skin temperature during an acute 
attack as well as inflammation and tissue destruction caused by deposition of monosodium urate crystals in the joints and other tissues (Roddy and Choi, 2014). Previous studies have shown that gout is an independent risk factor for chronic kidney disease (CKD). In one study, 24\% gout patients presented with CKD stage $\geq 3$ (Roughley et al., 2015). A systematic review found that the risk of developing kidney disease in patients diagnosed with gout was three times higher than in patients not diagnosed with gout (Kuo et al., 2016). Furthermore, CKD often progresses to end-stage renal disease, which is associated with premature death in gout patients (Kuo and Luo, 2017).

The goal of gout treatment in the acute stage of gouty arthritis mainly focuses on relieving pain while slowing down or stopping progression and further gout flares in the chronic stage, ultimately improving joint function ( $\mathrm{Lu}$ et al., 2020) and increasing the quality of life. Furthermore, it is important to reduce the incidence of gout-related CKD.

Drugs such as nonsteroidal anti-inflammatory drugs (NSAIDs), colchicine, and corticosteroids are recommended to alleviate severe pain during an acute gout attack. These drugs are effective for short-term management; however, their long-term use can cause adverse effects such as gastrointestinal reactions, rashes, and even renal failure (Smith et al., 2011; Perez-Ruiz et al., 2015; Smith et al., 2011; Perez-Ruiz et al., 2015). Moreover, such drugs cannot prevent or reverse the progression of this chronic metabolic disease. The current international guidelines recommend the use of xanthine oxidase inhibitors (such as allopurinol and febuxostat) as first-line treatment and uricosuric agents (such as benzbromarone) as second-line treatment for chronic gout. Nevertheless, allopurinol causes allergic reactions among Asians (Chen et al., 2019), febuxostat is associated with increased cardiovascular risk (Jutkowitz et al., 2014), and benzbromarone causes liver failure (Haring et al., 2013). Although several studies have been conducted on gout, the current treatment drugs are not satisfactory since all uratelowering drugs have potential side effects and drug interactions. Therefore, there is an urgent need for clinical treatments or drugs with high safety and good efficacy in lowering the concentration of uric acid to prevent the occurrence and progression of gout.

Traditional Chinese medicine (TCM) has a unique theoretical system and has shown significant clinical efficacy in the treatment of gout. Over the past few decades, extensive clinical trials have been conducted, especially in China (Chen, 2014; Xiao et al., 2018; Chi et al., 2020), to evaluate the use of Chinese herbal medicine (CHM) in the treatment of gout. The basic principle of Chinese medicine is syndrome differentiation and treatment. Under this guidance, classical CHM formulas and agents, such as Simiao San, Modified Simiao San, Gout decoction, and Danxi Gout decoction, are widely used in the treatment of damp-heat syndrome in gout patients, achieving good clinical efficacy (Chen, 2014; Xiao et al., 2018; Chi et al., 2020). In a previous study comparing the therapeutic advantages of herbs used in CHM therapy and chemical drugs such as colchicine, etoricoxib, and celecoxib in gout treatment, CHM therapy showed better effect in relieving acute pain, reducing the rate of recurrence, and lowering the concentration of uric acid with fewer side effects (Chen, 2014;
Xiao et al., 2018; Chi et al., 2020). Furthermore, (Chen, 2014; Xiao et al., 2018; Chi et al., 2020) found that a combination of CHM formulas and chemical drugs was better than the use of chemical drugs alone. However, there are safety concerns (such as hepatotoxicity, cardiotoxicity, and nephrotoxicity) with use of CHM therapy (Hong et al., 2006; Yang et al., 2007; Yang et al., 2012; Pan et al., 2020), severely hampering the clinical application and development of CHM therapies (Hong et al., 2006; Yang et al., 2007; Yang et al., 2012; Pan et al., 2020). Therefore, doctors and patients are seriously concerned that CHM therapy may increase the risk of CKD in gout treatment.

To our knowledge, no large-scale study has evaluated the association between CHM therapy and the incidence of CKD in gout patients. Thus, this study aimed to provide some evidence regarding the relationship between $\mathrm{CHM}$ therapy and the occurrence of CKD in gout patients.

\section{METHODS}

\section{Data Sources}

All research data were collected from the National Health Insurance Research database (NHIRD) of Taiwan from January 1, 1999 to December 31, 2013, and a total of One million people were randomly selected from the 23 million people included in the database Figure 1. The random sampling method was used by assigning serial numbers to 23 million beneficiaries in the sample population, and a random number generator was used to generate One million random values. To confirm the representativeness of the sample, we ensured that the age and sex distribution were not statistically different from that in the national sample. The NHIRD includes data on patient demographics, date of birth, sex, disease diagnoses, number of clinical visits and hospitalizations, and prescribed medications (with dosages), including CHM therapies administered by licensed TCM physicians. According to Taiwan Medical Law provisions, practicing physicians, including licensed TCM physicians, must be trained at a hospital or clinic designated by the central health authority for $>2$ years and obtain certification documents before commencing their individual practice. In Taiwan, licensed TCM physicians are required to make diagnoses using the International Classification of Diseases, Ninth Revision, Clinical Modification (ICD-9-CM) coding for claims. All diagnoses in this study were coded according to the ICD-9-CM.

\section{Participants}

A total of One million subjects were randomly selected from the NHIRD. In total, 24,311 patients aged $\geq 20$ years who were newly diagnosed with gout (ICD-9-CM code: 274) with least three outpatient visits or one hospitalization from January 1, 1999 to December 31, 2013 were selected in this study. Patients $(n=$ 716) who had been diagnosed with CKD before the first diagnosis of gout were excluded. 
TABLE 1 | Demographic characteristics of CKD and non-CKD.

\begin{tabular}{|c|c|c|c|c|c|}
\hline & \multicolumn{2}{|c|}{$\begin{array}{c}\text { CKD } \\
(N=1718)\end{array}$} & \multicolumn{3}{|c|}{ Non-CKD $(N=1,718)$} \\
\hline & $n$ & $\%$ & $n$ & $\%$ & $p$ value \\
\hline Age & & & & & 1 \\
\hline 20-64 & 597 & 34.7 & 597 & 34.7 & \\
\hline$\geq 65$ & 1121 & 65.3 & 1121 & 65.3 & \\
\hline Mean \pm SD & \multicolumn{2}{|c|}{$68.3 \pm 12.2$} & \multicolumn{2}{|c|}{$68.3 \pm 12.2$} & 1 \\
\hline Sex & & & & & 1 \\
\hline Female & 472 & 27.5 & 472 & 27.5 & \\
\hline Male & 1246 & 72.5 & 1246 & 72.5 & \\
\hline Hypertension & 1480 & 86.1 & 1201 & 69.9 & $<0.001$ \\
\hline Hyperlipidemia & 952 & 55.4 & 843 & 49.1 & $<0.001$ \\
\hline Chronic liver disease & 545 & 31.7 & 490 & 28.5 & 0.041 \\
\hline Diabetes & 797 & 46.4 & 560 & 32.6 & $<0.001$ \\
\hline COPD & 469 & 27.3 & 387 & 22.5 & 0.001 \\
\hline Autoimmune disease & 170 & 9.9 & 163 & 9.5 & 0.686 \\
\hline Cardiovascular disease & 725 & 42.2 & 616 & 35.9 & $<0.001$ \\
\hline Stroke & 479 & 27.9 & 341 & 19.8 & $<0.001$ \\
\hline Traditional Chinese medicine & 1049 & 61.1 & 1064 & 61.9 & 0.599 \\
\hline Corticosteroids & 907 & 52.8 & 668 & 38.9 & $<0.001$ \\
\hline Statin & 825 & 48.0 & 598 & 34.8 & $<0.001$ \\
\hline NSAIDs & 1634 & 95.1 & 1620 & 94.3 & 0.286 \\
\hline Aspirin & 925 & 53.8 & 752 & 43.8 & $<0.001$ \\
\hline Gout year & & & & & 1 \\
\hline 2000 & 895 & 52.1 & 895 & 52.1 & \\
\hline 2001 & 403 & 23.5 & 403 & 23.5 & \\
\hline 2002 & 296 & 17.2 & 296 & 17.2 & \\
\hline 2003 & 124 & 7.2 & 124 & 7.2 & \\
\hline
\end{tabular}

Bold font indicates statistical significance $(\mathrm{p}<0.05)$.

CKD, Chronic kidney disease; COPD, Chronic obstructive pulmonary disease; NSAIDs, Nonsteroidal anti-inflammatory drugs.

The patients with gout with CKD were categorized into the CKD group, while those without $\mathrm{CKD}$ were categorized into the non-CKD group. The CKD group included 2,000 patients who were diagnosed with CKD (ICD-9-CM code: 585) with at least three outpatient visits or one hospitalization 5 years after the diagnosis of gout. The non-CKD group comprised 20,006 patients who had not been diagnosed with CKD within 5 years after the diagnosis of gout.

The index date was set at 5 years after diagnosis with gout for both groups. The ratio of patients in the CKD group to those in the non-CKD group was 1:1; all subjects were matched in terms of age, sex, and gout diagnosis year.

\section{Chinese Herbal Medicine and Covariates}

The use of CHM was estimated from the first diagnosis of gout to the index date, and cumulative days of CHM therapy were used to calculate the dose effect. The main independent variables for CHM therapy among gout patients were selected from a series of demographic factors. Patients were classified into two groups according to age ( $<65$ years and $\geq 65$ years). Then, gout-associated diseases and treatments records were searched from the NHIRD database to identify independent variables. The baseline covariates included hypertension (ICD-9-CM codes: 401-405), hyperlipidemia (ICD-9-CM codes: 272.0-272.4), chronic liver disease (ICD-9-CM code: 571), diabetes (ICD-9-CM code: 250), chronic obstructive pulmonary disease (COPD; ICD-9-CM codes: 491, 492, and 496), autoimmune disease (ICD-9-CM codes: 710, 714, and 720), cardiovascular disease (ICD-9-CM codes: 410-414), and stroke (ICD-9-CM codes: 430-438). These covariates were identified based on at least three outpatient visits or one hospitalization for them between gout diagnosis and the index date. In addition, use of corticosteroids, statins, NSAIDs, and aspirin for a minimum of 30 days during the study period was evaluated.

\section{Statistical Analysis}

Student's t-test and the chi-square test were used to compare continuous and dichotomous variables, respectively, between CKD and non-CKD groups, and multivariate conditional logistic regression analysis was performed to investigate the risk of $\mathrm{CKD}$ in gout patients using CHM therapy after controlling for related confounding factors. SPSS version 18.0 (SPSS Inc, Chicago, IL, United States) was used for statistical analysis, and a $P$ value of $<0.05$ was considered statistically significant.

\section{RESULTS}

\section{Demographic Characteristics of Gout Patients in the Chronic Kidney Disease and Non-Chronic Kidney Disease Groups}

The demographic characteristics of gout patients in the CKD and non-CKD groups are shown in Table 1; the baseline characteristics were balanced between the groups. A total of 3,436 newly diagnosed gout patients were recruited from the claims database, including 1,718 patients in the CKD group and 1,718 patients in the non-CKD groups. The average age of the participants was approximately 68.3 years, with $34.7 \%$ patients aged $20-64$ years and the remaining $65.3 \%$ patients aged $\geq 65$ years. Among the enrolled patients, there were $27.5 \%$ female patients and $72.5 \%$ male patients. A total of 61.1 and $61.9 \%$ patients in the CKD and non-CKD groups were treated with $\mathrm{CHM}$, respectively.

In this study, the incidence of hypertension, hyperlipidemia, chronic liver disease, diabetes, COPD, cardiovascular disease, and stroke was significantly higher in the CKD group than in the non-CKD group. Furthermore, patients in the CKD group received more corticosteroids, statins, and aspirin than those in the non-CKD group. The proportion of patients with hypertension (86.1 vs. $69.9 \%$ ), hyperlipidemia (55.4 vs. $49.1 \%)$, chronic liver disease $(31.7$ vs. $28.5 \%$ ), diabetes ( 46.4 vs. $32.6 \%)$, COPD (27.3 vs. $22.5 \%)$, cardiovascular disease ( 42.2 vs. $35.9 \%)$, and stroke (27.9 vs. $19.8 \%$ ) was higher in the CKD group than in the non-CKD group (Table 1). Moreover, patients in the CKD group received more glucocorticoids (52.8 vs. $38.9 \%)$, statins ( 48.0 vs. $34.8 \%)$, and aspirin (53.8 vs. $43.8 \%$ ) than those in CKD group. However, there was no significant difference in the proportion of patients with autoimmune disease between the groups. Similarly, there was no significant difference in the proportion patients receiving CHM treatment between the groups. 
TABLE 2 | Conditional logistic regression of risk of CKD (1).

\begin{tabular}{|c|c|c|c|c|c|c|}
\hline & Crude OR & 95\% C.I. & $p$ value & Adjusted OR ${ }^{a}$ & 95\% C.I. & $p$ value \\
\hline $\mathrm{CHM}$ & 0.96 & $0.84-1.11$ & 0.593 & 1.01 & $0.86-1.18$ & 0.920 \\
\hline Hypertension & 3.04 & $2.50-3.68$ & $<0.001$ & 2.50 & 2.03-3.09 & $<0.001$ \\
\hline Hyperlipidemia & 1.30 & $1.13-1.49$ & $<0.001$ & 0.84 & $0.70-0.998$ & 0.047 \\
\hline Chronic liver disease & 1.17 & $1.01-1.36$ & 0.037 & 1.09 & $0.92-1.28$ & 0.308 \\
\hline Diabetes & 1.83 & $1.59-2.12$ & $<0.001$ & 1.48 & $1.26-1.73$ & $<0.001$ \\
\hline COPD & 1.32 & $1.12-1.55$ & 0.001 & 1.02 & $0.85-1.22$ & 0.810 \\
\hline Autoimmune disease & 1.05 & $0.84-1.31$ & 0.690 & 0.98 & $0.77-1.25$ & 0.862 \\
\hline Cardiovascular disease & 1.33 & $1.15-1.54$ & $<0.001$ & 0.96 & $0.81-1.14$ & 0.665 \\
\hline Stroke & 1.58 & $1.34-1.85$ & $<0.001$ & 1.24 & $1.04-1.49$ & 0.019 \\
\hline Corticosteroids & 1.75 & $1.52-2.01$ & $<0.001$ & 1.68 & $1.44-1.95$ & $<0.001$ \\
\hline Statin & 1.78 & $1.54-2.05$ & $<0.001$ & 1.49 & $1.24-1.79$ & $<0.001$ \\
\hline NSAIDs & 1.18 & $0.87-1.59$ & 0.286 & 0.96 & $0.69-1.33$ & 0.790 \\
\hline Aspirin & 1.58 & $1.37-1.83$ & $<0.001$ & 1.06 & $0.89-1.27$ & 0.504 \\
\hline
\end{tabular}

Bold font indicates statistical significance $(\mathrm{p}<0.05)$.

CHM, Chinese herb medicine; COPD, Chronic obstructive pulmonary disease; NSAIDs, Nonsteroidal anti-inflammatory drugs.

${ }^{a}$ Adjusted for hypertension, hyperlipidemia, chronic liver disease, diabetes, COPD, autoimmune disease, cardiovascular disease, stroke, corticosteroids, statin, NSAIDs, and aspirin.

\section{Chinese Herbal Medicine Use did Not Increase the Risk of Developing Chronic Kidney Disease}

Conditional logistic regression analysis was used to assess the risk factors of $\mathrm{CKD}$, including covariates such as CHM use; common complications such as hypertension, hyperlipidemia, chronic liver disease, and diabetes, and some chemical drugs. At the 5 years follow-up, CHM use did not increase the risk of developing CKD (Table 2) since there was no relationship between the incidence of CKD in gout patients and CHM use (adjusted odds ratio $[\mathrm{OR}]=1.01 ; 95 \%$ confidence interval $[\mathrm{CI}]$ : $0.86-1.18 ; p=0.920)$.

Hypertension (adjusted OR $=2.50 ; 95 \%$ CI: 2.03-3.09; $P<$ 0.001 ), diabetes (adjusted OR $=1.48 ; 95 \% \mathrm{CI}: 1.26-1.73 ; P<$ 0.001 ), and stroke (adjusted $\mathrm{OR}=1.24 ; 95 \% \mathrm{CI}: 1.04-1.49 ; P=$ 0.019) were associated with a higher risk of developing CKD. However, hyperlipidemia (adjusted OR $=0.84 ; 95 \% \mathrm{CI}$ : 0.70-0.998; $P=0.047$ ), chronic liver disease (adjusted $\mathrm{OR}=$ 1.09; 95\% CI: 0.92-1.28; $P=0.308$ ), COPD (adjusted OR $=1.02$; 95\% CI: 0.85-1.22; $P=0.810$ ), autoimmune disease (adjusted OR $=0.98 ; 95 \%$ CI: $0.77-1.25 ; P=0.862$ ), and cardiovascular disease (adjusted OR $=0.96 ; 95 \% \mathrm{CI}: 0.81-1.14 ; P=0.665)$ did not increase the risk of developing CKD. Furthermore, the use of corticosteroid (adjusted OR $=1.68$; 95\% CI: 1.44-1.95; $P<0.001$ ) and statin (adjusted OR $=1.49 ; 95 \% \mathrm{CI}: 1.24-1.79 ; P<0.001$ ) increased the risk of CKD, whereas the use of NSAIDs (adjusted $\mathrm{OR}=0.96 ; 95 \% \mathrm{CI}: 0.69-1.33 ; P=0.790$ ) and aspirin (adjusted OR $=1.06$; 95\% CI: $0.89-1.27 ; P=0.504)$ did not increase the risk of CKD.

\section{Chinese Herbal Medicine Use did Not Increase the Risk of Developing Chronic Kidney Disease in Different Sub-Populations}

Conditional logistic regression analysis was used to evaluate the association between CHM use and the risk of developing CKD in gout patients according to age and sex. Further stratification analysis according to different age groups ( $<65$ years vs. $\geq 65$ years) and sex (female vs. male) showed that there was no significant difference between the sub-populations (Table 3 ).

As shown in Table 3, the age of 1,194 and 2,242 patients was $<65$ and $\geq 65$ years, respectively. Of the 777 patients aged $<65$ years who received CHM therapy, 380 patients did not develop CKD (adjusted OR $=1.01 ; 95 \%$ CI: 0.77-1.34; $P<$ 0.05). However, of the 1,336 patients aged $\geq 65$ years who received CHM therapy, 669 patients did not develop CKD (adjusted OR $=1.03 ; 95 \%$ CI: $0.85-1.24 ; P<0.05$ ).

Moreover, of the 652 female patients who received CHM therapy, 323 patients did not develop CKD (adjusted OR = 1.04; 95\% CI: $0.75-1.45 ; P<0.05$ ), and of the 1,461 male patients who received CHM therapy, 726 patients did not develop CKD (adjusted OR = 1.00; 95\% CI: 0.84-1.19; $P<0.05$ ).

\section{Long-Term Use of Chinese Herbal Medicine did Not Increase the Incidence of Chronic Kidney Disease in Gout Patients}

Conditional logistic regression analysis did not reveal an association between cumulative days of CHM therapy (<90 days, 90-180 days, 180-365 days, $\geq 365$ days) and the incidence of $\mathrm{CKD}$ in gout patients (Table 4) even after $\geq 365$ days (adjusted OR $=1.30 ; 95 \%$ CI: 0.90-1.88).

\section{Top 10 Used Chinese Herbal Medicine Formulas}

Table 5 shows the top 10 used CHM formulas used in this study. They were Shujing Huoxue decoction (3.6\%), Duhuo Jisheng decoction (2.4\%), Jisheng Shenqi pill (2.2\%), Shaoyao Gancao decoction, Danggui Niantong decoction, Xuefu Zhuyu decoction, Liuwei Dihuang pill, Ganlu Yin, Jiawei Xiaoyao powder, and Chuanxiong Chatiao powder. Besides, the compositions of the top 20 formulas have been put in the supplementary document (Supplementary Table S1). 
TABLE 3 | Conditional logistic regression of risk of CKD (2).

\begin{tabular}{|c|c|c|c|c|c|c|c|c|}
\hline & $N$ & $\begin{array}{c}\text { No. } \\
\text { of CKD }\end{array}$ & Crude OR & 95\% C.I. & $\begin{array}{c}p \\
\text { value }\end{array}$ & $\begin{array}{l}\text { Adjusted } \\
\text { OR }^{\mathrm{a}}\end{array}$ & 95\% C.I. & $\begin{array}{c}p \\
\text { value }\end{array}$ \\
\hline \multicolumn{9}{|c|}{ Age <65 } \\
\hline \multicolumn{9}{|l|}{$\mathrm{CHM}$} \\
\hline No & 417 & 217 & 1 & & & 1 & & \\
\hline Yes & 777 & 380 & 0.88 & $0.69-1.12$ & 0.297 & 1.01 & $0.77-1.34$ & 0.923 \\
\hline \multicolumn{9}{|c|}{ Age $\geq 65$} \\
\hline \multicolumn{9}{|l|}{$\mathrm{CHM}$} \\
\hline No & 906 & 452 & 1 & & & 1 & & \\
\hline Yes & 1336 & 669 & 1.01 & $0.85-1.2$ & 0.930 & 1.03 & $0.85-1.24$ & 0.783 \\
\hline \multicolumn{9}{|c|}{ Female } \\
\hline \multicolumn{9}{|l|}{$\mathrm{CHM}$} \\
\hline No & 292 & 149 & 1 & & & 1 & & \\
\hline Yes & 652 & 323 & 0.94 & $0.70-1.25$ & 0.658 & 1.04 & $0.75-1.45$ & 0.801 \\
\hline \multicolumn{9}{|l|}{ Male } \\
\hline \multicolumn{9}{|l|}{$\mathrm{CHM}$} \\
\hline No & 1031 & 520 & 1 & & & 1 & & \\
\hline Yes & 1461 & 726 & 0.97 & $0.83-1.14$ & 0.714 & 1.00 & $0.84-1.19$ & 0.997 \\
\hline
\end{tabular}

CHM, Chinese herb medicine; CKD: Chronic kidney disease.

${ }^{a}$ Adjusted for hypertension, hyperlipidemia, chronic liver disease, diabetes, COPD, autoimmune disease, cardiovascular disease, stroke, corticosteroids, statin, NSAIDs, and aspirin.

TABLE 4 | Conditional logistic regression of risk of CKD (3).

\begin{tabular}{|c|c|c|c|c|c|c|c|c|}
\hline & $N$ & $\begin{array}{c}\text { No. } \\
\text { of CKD }\end{array}$ & Crude OR & 95\% C.I. & $p$-value & $\begin{array}{l}\text { Adjusted } \\
\text { OR }^{\mathrm{a}}\end{array}$ & 95\% C.I. & $p$-value \\
\hline \multicolumn{9}{|c|}{ Cumulative days of $\mathrm{CHM}$} \\
\hline None & 1323 & 669 & 1 & & & 1 & & \\
\hline$<90$ days & 977 & 476 & 0.92 & $0.79-1.07$ & 0.259 & 0.98 & $0.83-1.16$ & 0.821 \\
\hline \multirow[t]{2}{*}{ 90-180 days } & 338 & 157 & 0.90 & $0.68-1.19$ & 0.456 & 0.84 & $0.62-1.14$ & 0.266 \\
\hline & 798 & 416 & 1.33 & $0.98-1.8$ & 0.069 & 1.28 & $0.92-1.79$ & 0.143 \\
\hline \multicolumn{9}{|l|}{ 180-365 days } \\
\hline$\geq 365$ days & 3436 & 1718 & 1.17 & $0.83-1.64$ & 0.377 & 1.30 & $0.90-1.88$ & 0.162 \\
\hline
\end{tabular}

CHM, Chinese herb medicine; CKD, Chronic kidney disease.

${ }^{a}$ Adjusted for hypertension, hyperlipidemia, chronic liver disease, diabetes, COPD, autoimmune disease, cardiovascular disease, stroke, corticosteroids, statin, NSAIDs, and aspirin.

\section{DISCUSSION}

To our knowledge, this is the first study to investigate the correlation between the use of CHM and the risk of developing CKD in gout patients. The results showed that there was no association between CHM use and the occurrence of CKD gout patients.

Gouty arthritis is a chronic metabolic disease caused by abnormal purine metabolism and elevated serum uric acid concentrations, resulting in the deposition of urate crystals in the joints, kidneys, and other tissues (Lu et al., 2014). Therefore, patients with gout usually have comorbidities such as CKD. According to the United States National Health and Nutrition Examination Survey, the incidence of CKD of stage $\geq 2$ in gout patients was $71 \%$ (Zhu et al., 2012). In a previous study, $24 \%$ gout patients had CKD stage $\geq 3$ (Hong et al., 2006; Yang et al., 2007; Yang et al., 2012; Pan et al., 2020). Furthermore, according to a large population-based cohort study in the United Kingdom, compared to subjects without gout, those with gout had a $78 \%$ higher risk of developing CKD stage $\geq 3$ within 3 years of diagnosis (Roughley et al., 2018). Furthermore, CKD is expected to become one of the five main causes of death by 2040 , with enhanced risks of progression to end-stage renal disease and cardiovascular mortality (Foreman et al., 2018; Ortiz et al., 2019). Therefore, reducing the incidence of CKD or slowing down its progression in gout patients should be considered more seriously.

In this study, the incidence of hypertension, chronic liver disease, diabetes, COPD, cardiovascular disease, and stroke was significantly higher in the CKD group than in the non-CKD group. These diseases may be part of a series of clinical syndromes caused by impaired renal function, including metabolic syndrome and electrolyte imbalance. As established, the kidney is a significant target organ of serum urate; it has important endocrine and metabolic functions. Therefore, patients with hypertension, diabetes, 
TABLE 5 | The top 20 used Chinese medicine formulas.

\begin{tabular}{lcc}
\hline Chinese medicine formula & Frequency & $\%$ \\
\hline Shujing Huoxue Decoction & 2574 & 3.6 \\
Duhuo Jisheng Decoction & 1744 & 2.4 \\
Jisheng Shenqi Pill & 1576 & 2.2 \\
Shaoyao Gancao Decoction & 1418 & 2.0 \\
Danggui Niantong Decoction & 1332 & 1.9 \\
Xuefu Zhuyu Decoction & 1275 & 1.8 \\
Liuwei Dihuang Pill & 1188 & 1.7 \\
Ganlu Yin & 1103 & 1.5 \\
Jiawei Xiaoyao Powder & 1073 & 1.5 \\
Chuanxiong Chatiao Powder & 1057 & 1.5 \\
Gegen Decoction & 1055 & 1.5 \\
Yinqiao Powder & 1043 & 1.5 \\
Maxing Shigan Decoction & 989 & 1.4 \\
Tianwang Buxin Dan & 981 & 1.4 \\
Longdan Xiegan Decoction & 981 & 1.4 \\
Banxia xiexin Decoction & 978 & 1.4 \\
Pingwei Powder (Pill) & 954 & 1.3 \\
Zhibai Dihuang Pill & 933 & 1.3 \\
MaziRen Pill & 930 & 1.3 \\
Zhigancao Decoction & 923 & 1.3 \\
\end{tabular}

or stroke have a higher risk of developing CKD than other individuals. Hypertension, stroke, and other diseases may cause abnormal vascular function and renal perfusion, resulting in vascular renal damage, while metabolic disorders such as hyperlipidemia and diabetes can further aggravate the burden of kidney function (Jankowski et al., 2021). In addition, corticosteroid use increases the risk of developing CKD, possibly due to water and sodium retention. Further, statins are excreted via the kidney, possibly increasing he incidence of adverse events. According to a large registry study, patients with cardiovascular diseases and CKD who receive fewer evidence-based therapies, such as statins, $\beta$-blockers, and antiplatelet medicine, may have higher mortality rates than other individuals (Washam et al., 2015).

In this study, the presence of hyperlipidemia appeared to be a protective factor against the risk of developing CKD among gout patients; this finding is contrary to that reported by previous studies. Some patients may have been diagnosed with hyperlipidemia and treated with lipid-lowering drugs for a short time, resulting in statistical error. However, the underlying mechanisms are unknown; hence, further studied are needed.

This population-based nested case-control cohort study, we clarified that our previous speculation that CHM therapy may harm the kidney was false, even in different age and sex sub-populations. CHM therapy has been used for the treatment of gout for many years. In recent years, CHM formulas are being modified or combined with chemical drugs to create gout regimens with fewer side effects, some of which have been regarded as promising treatments. Moreover, this study found that some Chinese medicine formulas have been widely used in gout patients, such as Shujing Huoxue decoction, Duhuo Jisheng decoction, and Jisheng Shenqi pill. Further, Simiao powder and modified

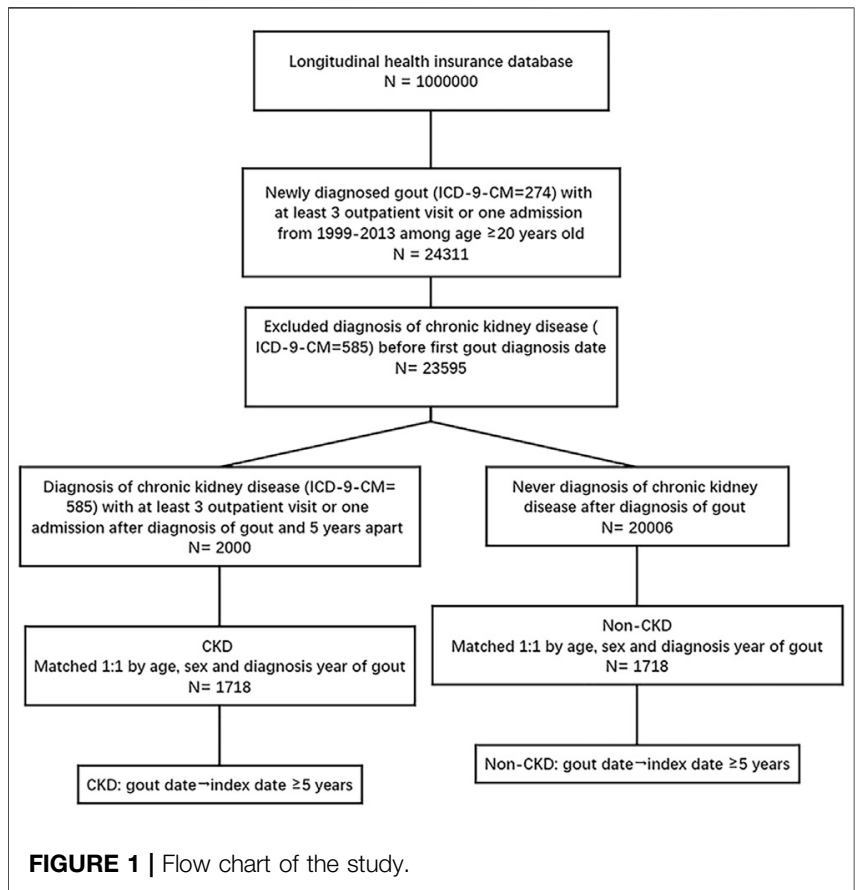

CHM formulas such as Jiawei Simiao powder, Tongfeng decoction, and Danxi Tongfeng decoction are widely used for treating damp-heat syndrome in gout patients. Chen (2014) found that the TCM formulas combined with chemical drugs showed better effects than chemical drugs alone, a finding similar to that reported by Huang et al. (2018). Therefore, CHM might be a promising safe therapy in treating gout.

In addition, CHM can also be used to treat a variety of kidney diseases. In recent years, an increasing number of scholars have conducted relevant studies on the efficacy and safety of CHM therapy in the treatment of acute kidney injury, chronic renal insufficiency, chronic glomerulonephritis, and other kidney diseases. In a study by Kuo-Chin Huang, CKD patients treated with TCM exhibited an increased long-term survival rate. Shen et al. (2018) reported that CHM formulas combine different herbal compounds to increase or promote therapeutic effectiveness, minimize toxicity and side effects, and optimize the therapeutic effects of each component, providing a safe and effective therapy for renal fibrosis. Therefore, the current study does not support that CHM therapy for gout can increase the risk of developing CKD. However, CHM therapy may delay disease progression in gout patients with $\mathrm{CKD}$ and may prolong the onset of CKD in gout patients without CKD.

\section{CONCLUSION}

This nested case-control study revealed that CHM therapy does not increase the incidence of CKD in gout patients, which clarifies our previous conjecture that traditional CHM may cause kidney damage. Further prospective clinical trials are needed to confirm our results. 


\section{DATA AVAILABILITY STATEMENT}

The raw data supporting the conclusion of this article will be made available by the authors, without undue reservation, to any qualified researcher.

\section{ETHICS STATEMENT}

The study was approved by the Institutional Review Board of the Chung Shan Medical University Hospital (CS17114).

\section{AUTHOR CONTRIBUTIONS}

Conceptualization, QX and JW; methodology, Y-HW; formal analysis, X-PC. and Y-HW; writing-original draft preparation,

\section{REFERENCES}

Chen, C. H., Chen, C. B., Chang, C. J., Lin, Y. J., Wang, C. W., Chi, C. C., et al. (2019). Hypersensitivity and Cardiovascular Risks Related to Allopurinol and Febuxostat Therapy in Asians: A Population-Based Cohort Study and Meta-Analysis. Clin. Pharmacol. Ther. 106 (2), 391-401. doi:10.1002/cpt. 1377

Chen, J. (2014). Clinical Effect of Jiawei Simiao Pills in the Treatment of Gouty Arthritis. J. Emerg. Tradit Chin. Med. 23 (03), 532-533.

Chi, X., Zhang, H., Zhang, S., and Ma, K. (2020). Chinese Herbal Medicine for Gout: a Review of the Clinical Evidence and Pharmacological Mechanisms. Chin. Med. 15, 17. doi:10.1186/s13020-020-0297-y

Foreman, K. J., Marquez, N., Dolgert, A., Fukutaki, K., Fullman, N., McGaughey, M., et al. (2018). Forecasting Life Expectancy, Years of Life Lost, and All-Cause and Cause-specific Mortality for 250 Causes of Death: Reference and Alternative Scenarios for 2016-40 for 195 Countries and Territories. The Lancet 392 (10159), 2052-2090. doi:10.1016/S01406736(18)31694-5

Haring, B., Kudlich, T., Rauthe, S., Melcher, R., and Geier, A. (2013). Benzbromarone. Eur. J. Gastroenterol. Hepatol. 25 (1), 119-121. doi:10. 1097/MEG.0b013e328358ace9

Hong, Y.-T., Fu, L.-S., Chung, L.-H., Hung, S.-C., Huang, Y.-T., and Chi, C.-S. (2006). Fanconi's Syndrome, Interstitial Fibrosis and Renal Failure by Aristolochic Acid in Chinese Herbs. Pediatr. Nephrol. 21 (4), 577-579. doi:10.1007/s00467-006-0017-6

Huang, K.-C., Su, Y.-C., Sun, M.-F., and Huang, S.-T. (2018). Chinese Herbal Medicine Improves the Long-Term Survival Rate of Patients with Chronic Kidney Disease in Taiwan: A Nationwide Retrospective Population-Based Cohort Study. Front. Pharmacol. 9, 1117. doi:10.3389/fphar.2018.01117

Jankowski, J., Floege, J., Fliser, D., Böhm, M., and Marx, N. (2021). Cardiovascular Disease in Chronic Kidney Disease. Circulation 143, 1157-1172. doi:10.1161/ CIRCULATIONAHA.120.050686

Jutkowitz, E., Choi, H. K., Pizzi, L. T., and Kuntz, K. M. (2014). Cost-effectiveness of Allopurinol and Febuxostat for the Management of Gout. Ann. Intern. Med. 161 (9), 617-626. doi:10.7326/M14-0227

Kuo, C.-F., Grainge, M. J., Mallen, C., Zhang, W., and Doherty, M. (2016). Comorbidities in Patients with Gout Prior to and Following Diagnosis: Case-Control Study. Ann. Rheum. Dis. 75 (1), 210-217. doi:10.1136/ annrheumdis-2014-206410

Kuo, C.-F., Grainge, M. J., Zhang, W., and Doherty, M. (2015). Global Epidemiology of Gout: Prevalence, Incidence and Risk Factors. Nat. Rev. Rheumatol. 11 (11), 649-662. doi:10.1038/nrrheum.2015.91

Kuo, C.-F., and Luo, S.-F. (2017). Risk of Premature Death in Gout Unchanged for Years. Nat. Rev. Rheumatol. 13 (4), 200-201. doi:10. 1038/nrrheum.2017.27
Y-ZX, Z-ZY, and Y-TL.; writing-review and editing, Y-ZX, Z-ZY, and Y-TL; supervision, QX. and JW; All authors have read and agreed to the published version of the manuscript.

\section{FUNDING}

This study was supported by the Natural Science Foundation of Guangdong Province (No. 2019A1515011636).

\section{SUPPLEMENTARY MATERIAL}

The Supplementary Material for this article can be found online at: https://www.frontiersin.org/articles/10.3389/fphar.2021.661282/ full\#supplementary-material

Lu, C.-C., Wei, J. C.-C., Chang, C.-A., Chen, C.-M., Tsai, S.-W., and Yeh, C.-J. (2020). Limited Knee-Joint Range of Motion in Patients with Tophaceous Gout Improved with Medical Treatment: A 18-Months Follow up. Front. Med. 7, 74 doi:10.3389/fmed.2020.00074

Lu, C.-C., Wu, S.-K., Chen, H.-Y., Chung, W.-S., Lee, M.-C., and Yeh, C.-J. (2014). Clinical Characteristics of and Relationship between Metabolic Components and Renal Function Among Patients with Early-Onset Juvenile Tophaceous Gout. J. Rheumatol. 41 (9), 1878-1883. doi:10. 3899/jrheum.131240

Mattiuzzi, C., and Lippi, G. (2020). Recent Updates on Worldwide Gout Epidemiology. Clin. Rheumatol. 39 (4), 1061-1063. doi:10.1007/s10067-01904868-9

Ortiz, A., Sanchez-Niño, M. D., Crespo-Barrio, M., De-Sequera-Ortiz, P., Fernández-Giráldez, E., García-Maset, R., et al. (2019). The Spanish Society of Nephrology (SENEFRO) Commentary to the Spain GBD 2016 Report: Keeping Chronic Kidney Disease Out of Sight of Health Authorities Will Only Magnify the Problem. Nefrología 39 (1), 29-34. English, Spanish. doi:10. 1016/j.nefro.2018.09.002

Pan, X., Zhou, J., Chen, Y., Xie, X., Rao, C., Liang, J., et al. (2020). Classification, Hepatotoxic Mechanisms, and Targets of the Risk Ingredients in Traditional Chinese Medicine-Induced Liver Injury. Toxicol. Lett. 323, 48-56. doi:10.1016/ j.toxlet.2020.01.026

Perez-Ruiz, F., Dalbeth, N., and Bardin, T. (2015). A Review of Uric Acid, Crystal Deposition Disease, and Gout. Adv. Ther. 32 (1), 31-41. doi:10.1007/s12325014-0175-z

Roddy, E., and Choi, H. K. (2014). Epidemiology of Gout. Rheum. Dis. Clin. North America 40 (2), 155-175. doi:10.1016/j.rdc.2014. 01.001

Roughley, M. J., Belcher, J., Mallen, C. D., and Roddy, E. (2015). Gout and Risk of Chronic Kidney Disease and Nephrolithiasis: Meta-Analysis of Observational Studies. Arthritis Res. Ther. 17 (1), 90. doi:10.1186/ s13075-015-0610-9

Roughley, M., Sultan, A. A., Clarson, L., Muller, S., Whittle, R., Belcher, J., et al. (2018). Risk of Chronic Kidney Disease in Patients with Gout and the Impact of Urate Lowering Therapy: a Population-Based Cohort Study. Arthritis Res. Ther. 20 (1), 243. doi:10.1186/s13075-018-1746-1

Shen, Y.-L., Wang, S.-J., Rahman, K., Zhang, L.-J., and Zhang, H. (2018). Chinese Herbal Formulas and Renal Fibrosis: An Overview. Cpd 24 (24), 2774-2781. doi:10.2174/1381612824666180829103355

Smith, H. S., Bracken, D., and Smith, J. M. (2011). Gout: Current Insights and Future Perspectives. The J. Pain 12 (11), 1113-1129. doi:10.1016/j.jpain.2011. 06.009

Washam, J. B., Herzog, C. A., Beitelshees, A. L., Cohen, M. G., Henry, T. D., Kapur, N. K., et al. (2015). Pharmacotherapy in Chronic Kidney Disease Patients Presenting with Acute Coronary Syndrome. Circulation 131, 1123-1149. doi:10.1161/CIR.0000000000000183 
Xiao, N., Chen, H., He, S.-Y., Xue, C.-X., Sui, H., Chen, J., et al. (2018). Evaluating the Efficacy and Adverse Effects of Clearing Heat and Removing Dampness Method of Traditional Chinese Medicine by Comparison with Western Medicine in Patients with Gout. EvidenceBased Complement. Altern. Med. 2018, 8591349. doi:10.1155/2018/ 8591349

Yang, L., Li, X., and Wang, H. (2007). Possible Mechanisms Explaining the Tendency towards Interstitial Fibrosis in Aristolochic Acid-Induced Acute Tubular Necrosis. Nephrol. Dial. Transplant. 22 (2), 445-456. doi:10.1093/ ndt/gfl556

Yang, L., Su, T., Li, X.-M., Wang, X., Cai, S.-Q., Meng, L.-Q., et al. (2012). Aristolochic Acid Nephropathy: Variation in Presentation and Prognosis. Nephrol. Dial. Transplant. 27 (1), 292-298. doi:10.1093/ndt/ gfr291
Zhu, Y., Pandya, B. J., and Choi, H. K. (2012). Comorbidities of Gout and Hyperuricemia in the US General Population: NHANES 2007-2008. Am. J. Med. 125 (7), 679-687. doi:10.1016/j.amjmed.2011.09.033

Conflict of Interest: The authors declare that the research was conducted in the absence of any commercial or financial relationships that could be construed as a potential conflict of interest.

Copyright $\odot 2021$ Xiao, Ye, Liang, Chen, Wang, Xu and Wei. This is an open-access article distributed under the terms of the Creative Commons Attribution License (CC BY). The use, distribution or reproduction in other forums is permitted, provided the original author(s) and the copyright owner(s) are credited and that the original publication in this journal is cited, in accordance with accepted academic practice. No use, distribution or reproduction is permitted which does not comply with these terms. 